\title{
Competencia y calidad de cartera en el mercado microfinanciero peruano, 2003-2015*
}

\author{
Ellen Mayorca Huamán ${ }^{*}$ \\ Giovanna Aguilar Andía**
}

\begin{abstract}
RESUMEN
El objetivo de este estudio es analizar la relación que existe entre la competencia y la calidad de cartera en el sector microfinanciero peruano en el periodo 2003-2015. Como indicador de competencia se emplea el poder de mercado estimado por el Índice de Lerner y como indicador de calidad de cartera se utiliza la tasa de morosidad. El análisis segmenta el mercado microfinanciero en tres grupos de instituciones microfinancieras (IMF), considerando el volumen promedio de sus colocaciones, de manera que, en el primer grupo se ubican las entidades con un mayor volumen promedio de colocaciones, en el segundo grupo se encuentran las entidades con un volumen medio de colocaciones y finalmente, en el tercer grupo se ubican las entidades con menor volumen promedio de colocaciones. Esta segmentación refleja la heterogeneidad de tamaño que existe entre estas entidades. Los resultados muestran un Índice de Lerner decreciente, para el primer y el tercer grupo, evidenciando una mayor competencia. En el segundo grupo, el comportamiento del Índice de Lerner muestra una tendencia creciente en los últimos meses del periodo estudiado, reflejando una menor competencia. Por otro lado, se evidencia una relación inversa entre el Índice de Lerner y la morosidad en los tres grupos, lo que implica que el aumento de competencia en el mercado microfinanciero ha generado un deterioro en la calidad de cartera crediticia. Este resultado se obtiene controlando el comportamiento de otras variables como son: el ciclo económico, la expansión de los créditos, la eficiencia y la rentabilidad de las instituciones, además del efecto de la crisis financiera internacional del 2008.
\end{abstract}

Palabras clave: microcrédito, microfinanzas, competencia, Índice de Lerner, morosidad, calidad de cartera.

Clasificación JEL: G21

\footnotetext{
* Este documento está basado en la Tesis de Licenciatura de Ellen Mayorca Huamán (2015), Departamento de Economía de la Pontificia Universidad Católica del Perú. Agradecemos los comentarios de los profesores Janina León (PUCP), Iván Rivera (PUCP) y Francisco Galarza (UP). Los errores y omisiones son de nuestra entera responsabilidad.

** Asistente de docencia e investigación en el Departamento de Economía de la Pontificia Universidad Católica del Perú (ellen.mayorcah@pucp.pe).

*** Profesora principal del Departamento de Economía de la Pontificia Universidad Católica del Perú (gaguila@pucp.edu.pe).
} 


\title{
Competition and loan portfolio quality in the Peruvian microfinance market, 2003-2015
}

\begin{abstract}
The aim of this study is to analyze the relationship between competition and loan portfolio quality in the Peruvian microfinance sector. To make this analysis the market is segmented into three groups of microfinance microfinance institutions IMFs), considering the average volume of its loans. So that, in the first group (large IMFs) entities of higher average volume of loans are located, in the second group (medium IMFs) entities with an average volume of loans are located and finally, in the third group (small IMFs) are entities with lower average loan volumes. This segmentation reflects the heterogeneity of size for loans between IMFs. The results show the Lerner index decreasing over time, for both the group of large IMFs and the group of small IMFs, showing a decrease of market power and increased competition in these two groups of entities. In the group of medium IMFs behavior Lerner index shows a growing trend in the last months of the period studied thus indicating a greater market power and less competition in this group of microfinance operators. On the other hand, an inverse relationship between the Lerner index over delinquency rate in the three groups of IMFs is found, implying that increased competition in the Peruvian microfinance market has deteriorated the quality of loan portfolio the period analyzed. This result is obtained by controlling the behavior of other important variables to explain the delinquency rate such as the business cycle, credit expansion, efficiency and profitability of institutions, and the effect of the international financial crisis of 2008.
\end{abstract}

Keywords: microcredit, microfinance, competition, Lerner index, delinquency rate, quality loan portfolio.

JEL Code: G21

\section{INTRODUCCIÓN}

En el mercado microfinanciero peruano operan cinco tipos de instituciones microfinancieras (IMF) reguladas por la Superintendencia de Banca Seguros y Administradoras de Fondos de Pensiones (SBS): las cajas municipales de ahorro y crédito (CMAC), las cajas rurales de ahorro y crédito (CRAC), las entidades de apoyo al desarrollo de pequeñas y microempresas (edpyme), las financieras y un banco especializados en microfinanzas.

Hace más de treinta años que se fundaron las primeras IMF, y desde entonces el desarrollo de estas instituciones y del mercado de microfinanzas peruano ha sido notable en términos de la expansión de la oferta de servicios microcrediticios y de ahorro dirigida a hogares de medios y bajos ingresos y a pequeńas y microempresas, segmento de la población tradicionalmente excluido de los servicios prestados por la banca comercial.

El crecimiento de las microfinanzas en el Perú ha conllevado a la maduración del mercado en un contexto de cambios regulatorios, así como del ingreso de la banca 
comercial al sector — downscaling - y al crecimiento y transformación de las propias IMF — upgrading - Estas transformaciones vienen generando presiones sobre la competencia en el mercado. En efecto, la presencia de la banca comercial, la llegada de las CMAC al mercado capitalino e importantes cambios en la escala de operación de algunas edpyme, están impulsando una mayor competencia en el mercado microfinanciero a nivel nacional. La reducción de tasas de interés activas así como, el ingreso a nuevas áreas geográficas por parte de las IMF y la apertura de nuevas oficinas, son señales de la intensificación de la competencia en el mercado.

Un aumento de competencia en el mercado microfinanciero puede generar distintos efectos sobre la calidad de cartera de las instituciones. Si la mayor competencia por clientes obliga al relajamiento de los controles de riesgo y exigencias en el otorgamiento del crédito, esto podría tener como consecuencia un deterioro de la calidad de cartera microcrediticia poniendo en peligro la estabilidad financiera de las entidades. Adicionalmente, la presión competitiva puede conducir a un sobreendeudamiento de los clientes, deteriorando aún más la calidad de la cartera. Por el contrario, si la mayor competencia incentiva a las entidades a ser más eficientes, la ganancia de eficiencia debería mejorar los indicadores financieros de las instituciones, entre ellos, la calidad de sus carteras crediticias. Por ello, a priori, no sería posible predecir el efecto que tiene la mayor competencia en el mercado microfinanciero peruano, por lo cual es necesario llevar a cabo un análisis empírico para determinar cuál de los dos efectos es el que prevalece.

El objetivo de este estudio es analizar la relación que existe entre la competencia y la calidad de cartera en el sector microfinanciero peruano en el periodo 2003-2015. Como indicador de competencia se emplea el poder de mercado, estimado mediante el Índice de Lerner y como indicador de calidad de cartera se utiliza la tasa de morosidad, que mide el cociente entre los créditos vencidos y los créditos totales. El análisis segmenta el mercado microfinanciero en tres grupos de IMF, considerando el volumen promedio de sus colocaciones de manera que, en el primer grupo - IMF grandes - se ubican las entidades con un mayor volumen promedio de créditos, en el segundo grupo, - IMF medianas - se encuentran las entidades con un volumen medio de colocaciones y finalmente, en el tercer grupo - IMF pequeñas - se ubican las entidades con menor volumen promedio de colocaciones. Esta segmentación refleja la heterogeneidad de tamaño de las IMF.

Si bien la banca comercial también ofrece microcréditos en el mercado, se ha desestimado su incorporación en este análisis debido a que su oferta está dirigida a un segmento de clientes distinto al atendido por las IMF, a juzgar por el valor del promedio del microcrédito. En efecto, a diciembre de 2015 el valor promedio del microcrédito ofrecido por la banca comercial ascendió a $S / 18000$ mientras que el microcrédito promedio de las CMAC, ascendió a S/5000. Así, la banca comercial atiende la demanda de los clientes microfinancieros de más altos ingresos mientras que las IMF de menores ingresos. 
Los resultados obtenidos muestran un Índice de Lerner decreciente a lo largo del periodo, tanto para el grupo de IMF grandes como para el grupo de IMF pequeñas, evidenciando una disminución del poder de mercado y una mayor competencia en estos dos grupos de entidades. En el grupo de IMF medianas, el comportamiento del Índice de Lerner muestra una tendencia creciente en los últimos meses del periodo estudiado indicando con ello, un mayor poder de mercado y una menor competencia en este grupo de operadores microfinancieros. Por otra parte, se evidencia una relación negativa entre el Índice de Lerner y la morosidad crediticia en los tres grupos de IMF, lo cual implica que el aumento de competencia en el mercado microfinanciero peruano ha generado un deterioro en la calidad de cartera crediticia en el periodo analizado. Este resultado se obtiene controlando el comportamiento de otras variables importantes para explicar la morosidad microcrediticia como son el ciclo económico, la expansión de los créditos, la eficiencia y la rentabilidad de las instituciones, además del efecto de la crisis financiera internacional del 2008.

El resto del documento se organiza de la siguiente manera: la sección 2 presenta el marco teórico del estudio, la sección 3 muestra una revisión de la literatura más actual sobre el tema de competencia y sus efectos en la calidad de cartera, la sección 4 presenta los principales hechos estilizados en torno a la competencia y la calidad de cartera en el mercado microcrediticio peruano. En la sección 5 se presenta la estrategia empírica a seguir para el análisis cuantitativo, mientras que en la sección 6 se exponen los resultados encontrados. Las principales conclusiones del estudio se encuentran en la sección 7.

\section{MARCO TEÓRICO}

Según la teoría neoclásica, un mercado logra estabilidad, eficiencia y maximiza el bienestar social si presenta una estructura de competencia perfecta. Sin embargo, este tipo de competencia no se alcanza en la mayoría de los mercados. Stiglitz y Welss (1981) señalan que el mercado financiero se caracteriza por ser uno de los mercados que más se aleja de la competencia perfecta porque presenta varias fallas de mercado ${ }^{1}$. A pesar de ello, en la actualidad se viene desarrollado una mayor competencia en los mercados financieros. Boone, Van Ours y Wiel (2007) mencionan que «la competencia puede ser intensificada en un mercado por: i) el ingreso de nuevas firmas en el mercado dada una reducción de las barreras de entrada y ii) una mayor conducta defensiva de las firmas incumbentes» [traducción propia]. Ambas condiciones se cumplen en el mercado financiero con el surgimiento de nuevas entidades financieras así como, con el crecimiento de grupos financieros que gracias a una conducta competitiva más activa, están adquiriendo una mayor cuota de mercado (Vives, 2014).

1 Estas fallas son información asimétrica, barreras de entradas y externalidades (Vives, 2014). 
La literatura relevante no ofrece un consenso sobre los efectos que genera una mayor competencia en el mercado financiero, sobre la calidad de la cartera crediticia de las entidades. Por un lado, existe la postura que relaciona positivamente la competencia con la calidad de cartera. Se presentan argumentos que afirman que una mayor presión competitiva incentiva a las firmas a ser más eficientes, reduciendo sus costos de producción (Boone, 2007). Asimismo, las firmas pueden desarrollar economías de escala y diversificar su oferta de productos y servicios (Assefa, Helmes y Meesters, 2013). La ganancia de eficiencia implicaría la reducción del riesgo en sus actividades, permitiendo una mejora en la calidad de cartera crediticia. En efecto, Hauswald y Márquez (2003) afirman que un contexto de mayor competencia, impulsa a los intermediarios financieros al desarrollo de nuevas tecnologías financieras de evaluación y control del riesgo crediticio lo que mejora la calidad de sus carteras crediticias. Asimismo, Caminal y Matutes (2002), Boyd y De Nicoló (2005) señalan que los bancos con mayor poder de mercado tienden a incurrir en un mayor riesgo crediticio al fijar elevadas tasas de interés a sus clientes, elevando con ello la probabilidad de no repago de los créditos.

Por otro lado, existe otra postura que relaciona negativamente, la competencia con la calidad de cartera. Según esta, un incremento de la competencia en el mercado incentiva a las entidades financieras a incorporar nuevos clientes de mayor riesgo y a retener a los antiguos, flexibilizando y/o disminuyendo sus controles de riesgo en el primer caso y a sobreendeudarlos, en el segundo (Gehrig, 1998; Hellman, Murdock y Stiglitz, 2000), lo cual termina deteriorando su calidad de cartera. Asimismo, una mayor competencia incentiva a las entidades a participar en operaciones más riesgosas para compensar la reducción de los márgenes de beneficios (Beck, 2008; Maudos y Fernández, 2010).

Además, en un mercado donde hay múltiples ofertantes habrá una mayor facilidad para cambiar de entidad o para refinanciar los créditos con la misma u otra(s) entidad(es), lo cual acrecentaría los problemas de riesgo moral (Barrón, 2011). Según Petersen y Rajan (1995), un banco con poder de mercado tiene una alta posibilidad de reducir el problema de asimetría de información al desarrollar adecuadamente sus relaciones con sus clientes. Esto les permite discriminar adecuadamente entre prestatarios de bajo riesgo y los de alto riesgo (Ceterelli y Peretto, 2000).

A priori, no es posible establecer de manera categórica si una mayor competencia en el mercado de créditos podría mejorar o deteriorar la calidad de cartera de las instituciones intermediarias por lo que es necesario realizar un análisis empírico y considerar las características particulares del mercado financiero que se desea analizar (Allen y Gale, 2004). Por ello es relevante considerar, en el análisis, otros factores que afectan a la calidad de cartera para realizar un análisis completo de su comportamiento.

La literatura relevante, considera determinantes de carácter macroeconómico y microeconómico. Entre los macroeconómicos se encuentran el ciclo económico, el nivel de endeudamiento general de los agentes, el tipo de cambio, entre otros (Aguilar y Camargo, 2004; Díaz, 2009). Mientras que, entre los determinantes microeconómicos 
se incluyen, el crecimiento de la cartera de créditos, la tasa de interés activa, la rentabilidad y el nivel de eficiencia con el que opera la entidad (Díaz, 2009; Vela y Uriol, 2012).

En relación con los determinantes macroeconómicos, el ciclo económico es un factor relevante para determinar la calidad de la cartera crediticia. Un ciclo expansivo, contribuye a mejorar la calidad de la cartera en la medida que eleva la probabilidad de repago de los créditos mientras que un ciclo recesivo o de desaceleración económica, genera un deterioro de la calidad de cartera de créditos al reducir la probabilidad de repago de créditos (Aparicio, Gutiérrez, Jaramillo y Moreno, 2013).

El nivel de endeudamiento de los agentes brinda señales sobre los riesgosos que son, en cuanto a la posibilidad de no cumplir con el pago de sus créditos. Un elevado nivel de endeudamiento de los agentes en una economía, estaría asociado con una mayor probabilidad de incumplimiento de repago y por tanto, con un deterioro de la calidad de la cartera crediticia. En la misma dirección, el tipo de cambio afecta a la calidad de cartera, especialmente cuando los créditos están, en su mayoría, denominados en moneda extranjera ya que un incremento del tipo de cambio puede generar una mayor carga de deuda para los agentes originando una disminución en el pago de los créditos (Bangia, Diebold y Schuermann, 2002; Jones, 2005).Una variable muy importante a considerar en el análisis es la ocurrencia de crisis financieras. En efecto, este tipo de eventos deterioran el contexto para el desarrollo de las actividades económicas de los agentes por lo que, estos presentan dificultades para cumplir con el pago de sus deudas, que pueden traducirse en un aumento de la morosidad de las carteras de créditos. En el Perú, la crisis financiera internacional de 2008, mostró sus primeras consecuencias sobre la actividad económica a partir de octubre de ese año.

Respecto a los factores microeconómicos, con la finalidad de defender su cuota de mercado, las entidades buscan incrementar los créditos que otorgan y muchas veces, para lograrlo relajan sus controles de riesgo, disminuyendo la calidad de su cartera crediticia (Aparicio et al., 2013). La tasa de interés cobrada por los créditos otorgados puede ser un determinante de la calidad de cartera si es que esta es demasiado elevada aumentando con ello la posibilidad de riesgo de impago (Hauswald y Marquez, 2003). El nivel de rentabilidad procurado por la institución puede afectar la calidad de la cartera de créditos pues, cuando por alcanzar elevados de rentabilidad la institución incurre en manejos poco cuidadosos de los niveles de riesgo de su cartera. La eficiencia de la gestión también es un factor que afecta la calidad de créditos. Cuando la entidad no gestiona adecuadamente su cartera crediticia, puede elevar sus niveles de incumplimiento deteriorando con ello la calidad de su cartera de créditos.

Por último, para evaluar la competencia en un mercado se han empleado indicadores como el índice de Herfindahl Hirschman (IHH), el estadístico H de Panzar y Rose, el Índice de Lerner, el indicador de Boone, entre otros. En la presente investigación, se emplea el Índice de Lerner como indicador de competencia porque puede calcularse a nivel de intermediario financiero y porque revela el poder que tiene cada institución para establecer un precio por encima de su costo marginal, es decir, revela su poder de mercado. 
En un mercado de competencia perfecta, el poder de mercado de los ofertantes es nulo mientras que, en mercados donde la competencia es reducida, el poder de mercado es mayor.

En cuanto a la calidad de cartera, el indicador utilizado convencionalmente para medirla es la tasa de morosidad, calculada como el cociente entre los créditos en situación de atraso y el total de la cartera crediticia. Una mayor tasa de morosidad estaría reflejando un mayor riesgo crediticio ${ }^{2}$ y por tanto una menor calidad de cartera. Un deterioro en la calidad de cartera, obliga a la entidad a incrementar sus provisiones por créditos impagos lo que reduce sus utilidades, deteriorándose así sus resultados financieros (Pérez, 2009).

\section{ESTADO DE LA CUESTIÓN}

La relación entre la competencia del mercado y la calidad de cartera de las instituciones financieras -bancarias y no bancarias- ha sido objeto de varios estudios. Entre las investigaciones más recientes que presentan evidencia de una relación negativa entre competencia y calidad de cartera se encuentran las de investigadores como Berger, Klapper y Turk-Ariss (2009), Turk (2010), Maudos y Fernández de Guevara (2010). Un reciente análisis aplicado al mercado microfinanciero es el de Kumar y Bali (2014), en el que también se encuentra evidencia según la cual, más competencia en el mercado deteriora la calidad de la cartera de los microcréditos. Por el contrario, estudios como los de Beck, Demirguc-Kunt y Levine (2006) y Shaeck, Cihak y Wolfe (2009) proporcionan resultados a favor de una relación positiva entre competencia y calidad de cartera.

En el caso específico del mercado microcrediticio peruano, Talledo (2014) analizó la relación entre la morosidad de los créditos a microempresas de las cajas rurales, y la competencia encontrando que cuando esta se intensifica se deteriora la calidad de la cartera crediticia.

Otros estudios han trabajado temas como el de la competencia en el mercado microfinanciero peruano (Del Pozo, 2008; Aguilar, 2014) o el de la calidad de cartera (Aguilar y Camargo, 2004; Vela y Uriol, 2012) pero sin analizar su relación.

\section{HECHOS ESTILIZADOS}

El origen de las IMF en el Perú se remonta a la década de 1980 con la creación de las primeras cajas municipales de ahorro y crédito (CMAC) y la aparición de organizaciones no gubernamentales $(\mathrm{ONG})$ con programas de microcrédito. Posteriormente, durante la década de 1990, surgirían las cajas rurales de ahorro y crédito (CRAC), luego de la desactivación de la banca de desarrollo agrario, como intermediarios especializados para atender

\footnotetext{
2 Se define al riesgo crediticio como el potencial riesgo de que el prestatario o contraparte de una institución financiera no se encuentre en capacidad de responder por las obligaciones que tiene con la institución (Comité de Basilea, 2000).
} 
las necesidades de financiamiento del sector rural (agropecuario). Asimismo, en la misma década se crearon las primeras entidades de desarrollo para la pequeña y microempresa (edpyme) como resultado de la formalización de programas de microcrédito de algunas ONG (Conger, Inga y Webb, 2009). Además de estas entidades, también se instauraron los bancos especializados en microfinanzas y después, las entidades financieras especializadas en microfinanzas, resultado del crecimiento y transformación de algunas edpyme.

El periodo de análisis de esta investigación va de 2003 a 2015. En este periodo se mejoró sustancialmente la regulación prudencial de las entidades financieras, a partir del fortalecimiento de la SBS y las pautas planteadas por el Comité de Basilea. Por otra parte, este periodo se caracteriza por la relativa estabilidad macroeconómica a pesar de los efectos de la crisis financiera de 2008.

Durante la primera década de este siglo, las microfinanzas en el Perú tuvieron un significativo crecimiento que llevó a la conformación de un mercado maduro y heterogéneo. En efecto, las IMF peruanas han mostrado incrementos notables de sus carteras de colocaciones y del número de sus clientes llevando a conformar un segmento del sistema financiero con instituciones de distinto tamaño y ámbito de acción. Asimismo, la heterogeneidad de las microfinanzas peruanas se debe, entre otros aspectos, a la distinta naturaleza institucional que tienen las IMF en el Perú. El dinamismo y crecimiento del sector se ha producido junto con la actuación de bancos especializados en microfinanzas y la transformación de las edpyme en instituciones financieras.

A diciembre del 2015, el sector microfinanciero peruano estuvo conformado por 38 IMF, de las cuales 12 eran CMAC, 7 eran CRAC, 12 edpyme, 7 financieras y un banco, especializados en microfinanzas.

Dada la heterogeneidad de IMF, mencionada líneas arriba, se ha considerado segmentar el conjunto de IMF en tres grupos de acuerdo al volumen promedio anual de colocaciones. El primer grupo - IMF grandes - está conformado por $10^{3}$ entidades cuyo volumen promedio anual de colocaciones es mayor a 400 millones de soles. En el segundo grupo - IMF medianas - se encuentran las firmas cuyo promedio anual de colocaciones se encuentran entre 400 millones y 100 millones de soles, siendo conformado por 12 entidades $^{4}$. El último grupo - IMF pequeñas - está conformado por 20 entidades $^{5}$ y son aquellas que tienen colocaciones, promedio, por debajo de 100 millones de soles.

El Gráfico 1 muestra que a diciembre del 2015, las IMF grandes concentraron el 78\% de las colocaciones del mercado microfinanciero, mientras que las medianas participaron con el $22 \%$ y las IMF pequeńas, lo hicieron con apenas el $2 \%$ del total colocaciones. Esta distribución de créditos refleja la heterogeneidad en el tamaño de las IMF que existe en el mercado microfinanciero peruano.

\footnotetext{
5 CMAC, 3 financieras, una CRAC y un banco especializado en microfinanzas.

4 CMAC, 3 CRAC, 3 edpyme y 2 financieras.

3 CMAC, 8 CRAC, 7 edpyme y 2 financieras.
} 


\section{Gráfico 1. Distribución de la colocaciones totales de las IMF} por grupos a diciembre del 2015

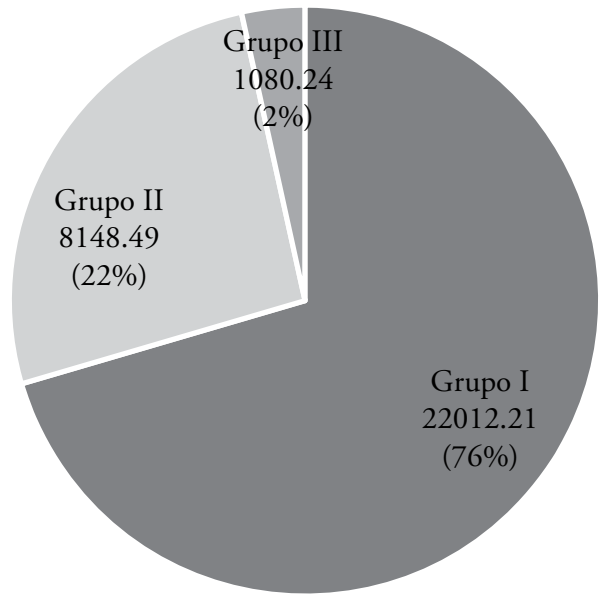

Fuente: SBS. Elaboración propia.

Durante el periodo analizado se ha registrado en el mercado microfinanciero una significativa reducción de tasas activas, tal como se aprecia en el Gráfico 2. Este comportamiento en el costo del microcrédito es en parte, resultado de la mayor competencia observada en el mercado microcrediticio peruano. Es importante mencionar también, que los promedios de tasas cobradas por las IMF más grandes son históricamente menores que las cobradas por IMF de menor tamaño (medianas y pequeñas) aun cuando se observa una tendencia a la convergencia hacia el final del periodo estudiado.

Gráfico 2. Tasa de interés activa promedio de créditos a microempresas

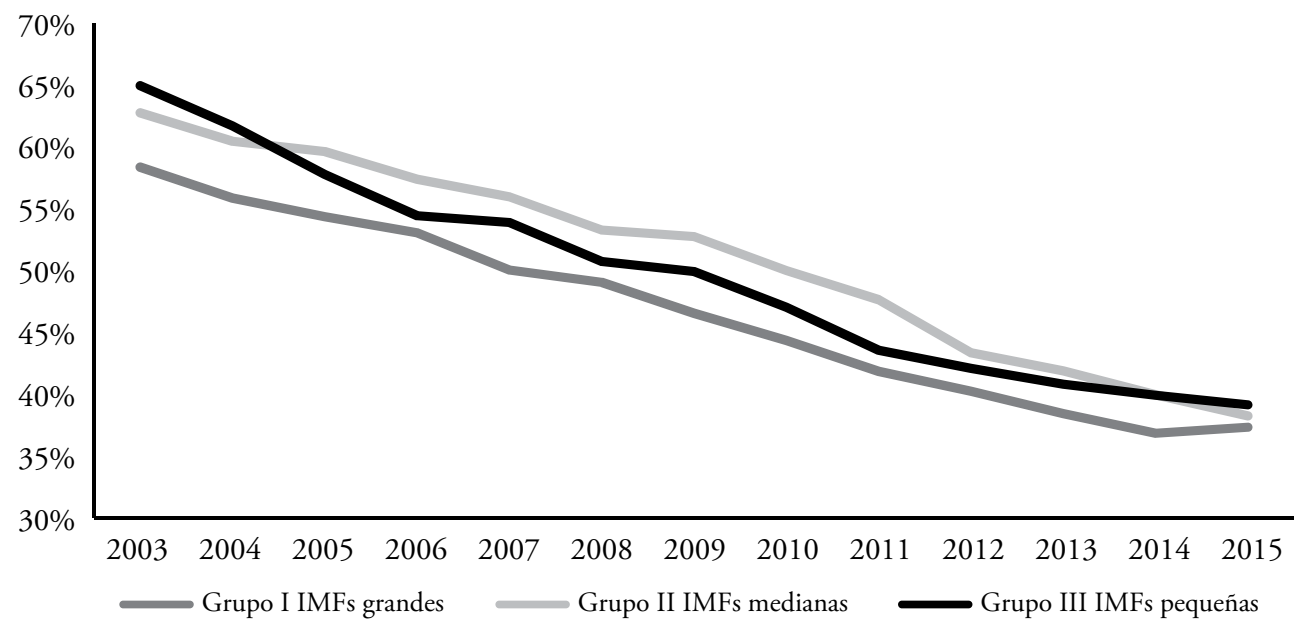

Fuente: SBS. Elaboración propia. 
Otro hecho estilizado consistente con la mayor competencia en el mercado microfinanciero, es la reducción de utilidades registrada en el periodo estudiado (ver Gráfico 3). Esto es principalmente cierto en el caso del grupo de IMF grandes. Lo que revelaría que la competencia se he profundizado más, en este grupo de intermediarios microfinancieros.

\section{Gráfico 3. Utilidades netas promedio}

(En miles de soles)

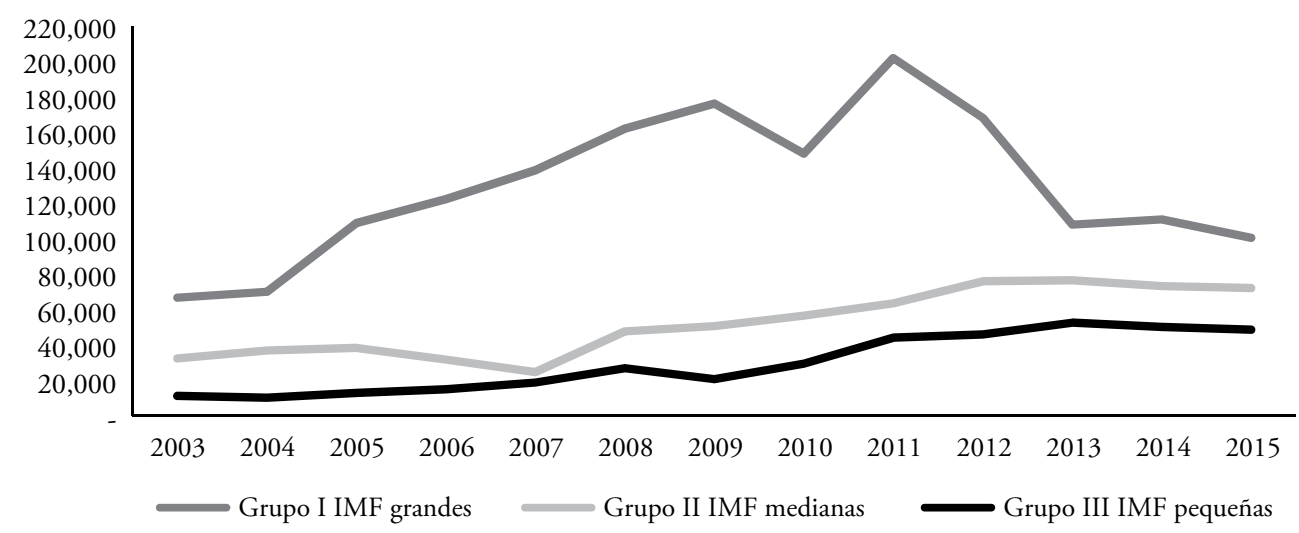

Fuente: SBS. Elaboracion propia.

Finalmente, se presenta la evolución de las tasas de morosidad promedio para los grupos de IMF en el Gráfico 4.

Gráfico 4. Tasa de morosidad promedio

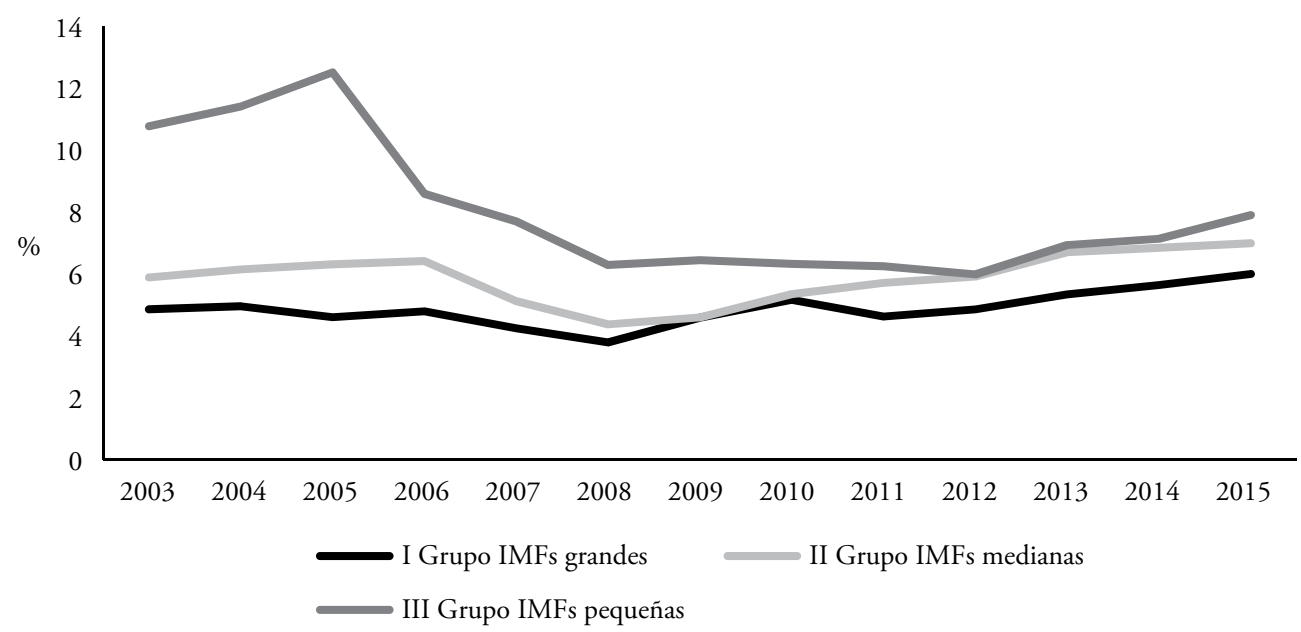

Fuente: SBS. 
Si bien la morosidad registró un descenso hasta antes de la crisis financiera internacional del año 2008, luego ha mostrado una tendencia creciente en todos los grupos de intermediarios financieros. Este deterioro de la cartera de créditos de las IMF en el periodo de análisis se ha producido en un contexto caracterizado por una mayor competencia en el mercado y la ocurrencia de una crisis financiera internacional por lo que este último acontecimiento tiene que ser controlado en el análisis empírico de la relación entre competencia y calidad de cartera como se verá más adelante.

\section{ESTRATEGIA EMPÍRICA}

El componente econométrico de la presente investigación tiene dos partes. En la primera, se procede a la estimación del Î́ndice de Lerner y en la segunda, a la estimación del efecto de la competencia en la calidad de cartera.

\subsection{ESTIMACIÓN DEL ÍNDICE DE LERNER}

El Índice de Lerner es calculado mediante la siguiente fórmula:

$$
I L=\frac{(P-C M)}{P}
$$

Donde $P$ es el precio del output y $C M$ es el costo marginal. El precio del output se calcula como el ratio entre los ingresos financieros y los activos totales (Maudos, Fernández y Pérez, 2002) mientras que el costo marginal se deriva de una función de costo total (CT). La estimación de una función de costo total exige la definición del output y los inputs en la actividad de intermediación financiera existiendo para ello, dos enfoques alternativos. Por un lado, el enfoque de producción establece que los intermediarios financieros producen depósitos y créditos usando como inputs la mano de obra y el capital. Bajo este enfoque, el output se mide en cantidades, es decir, número de cuentas de créditos y/o depósitos (Benston, 1965; Bell y Murphy, 1968). Por otro lado, el enfoque de intermediación define el output como los distintos tipos de créditos ofrecidos, y los inputs con los que estos se producen son la mano de obra, el capital físico y los recursos financieros o fondos prestables (Benston, Hanweck y Humphrey, 1982). De acuerdo con este enfoque, el output se mide como el valor de los distintos créditos ofrecidos.

Considerando las características del mercado microfinanciero, en el cual los costos financieros son muy relevantes en la estructura de costos de las IMF (Aguilar, 2014) se empleará el enfoque de intermediación. Según este enfoque, el costo total incorpora los costos financieros y operativos de las IMF mientras que el output representa la cartera de créditos. Los precios de los insumos de producción son los salarios, el costo del capital físico y el costo del capital financiero, es decir, los retornos a los factores de producción 
La función de costos translogarítmica a estimar es:

$$
\begin{aligned}
& \ln C_{i t}=\alpha_{0}+\sum_{j=1}^{3} \alpha_{j} \ln w_{i t}^{j}+\frac{1}{2} \sum_{j=1}^{3} \sum_{k=1}^{3} \alpha_{j k} \ln w_{i t}^{j} \ln w_{i t}^{k}+\beta_{0} \ln Y_{i t}+\frac{1}{2} \beta_{00} \ln Y_{i t}^{2} \\
& +\sum_{j=1}^{3} \beta_{0 j} \ln Y_{i t} \ln w_{i t}^{j}+\gamma_{t} T+\frac{1}{2} \gamma_{t t} T^{2}+\sum_{j=1}^{3} \gamma_{j t} T \ln w_{i t}^{j}+\gamma_{t 0} T \ln Y_{i t}+u_{i t}
\end{aligned}
$$

Donde $C_{i t}$ representa el costo total, $Y_{i t}$ representa el output, $w_{i t}^{j}$ representa el precio de cada factor de producción $(j=1,2,3)$. Además, se ha incorporado un componente tendencial $(T)$ para tomar en cuenta la existencia de cambio tecnológico. Los subíndices it indican la observación para la $i$-ésima entidad en el periodo $t$, respectivamente.

Esta función de costos translogarítmica debe cumplir con las siguientes propiedades para asegurar la homogeneidad lineal en precios de los factores y el Teorema de Young:

$$
\sum_{\mathrm{j}=1}^{3} \pm_{\mathrm{j}}=1 ; \sum_{\mathrm{j}=1}^{3} \pm_{\mathrm{jk}}=0 ; \sum_{\mathrm{i}=1}^{\mathrm{N}} \beta_{0 j}=0 ; \alpha_{j k}=\alpha_{k j} ; \beta_{00}=\beta_{00}
$$

A partir de la función de costos se obtiene el costo marginal $\left(\mathrm{Cmg}_{i t}\right)$ :

$$
C m g_{i t}=\left(\frac{C_{i t}}{y_{i t}}\right)\left(\beta_{0}+\beta_{00} \ln y_{i t}+\sum_{j=1}^{3} \beta_{0 j} \ln w_{j i t}+\gamma_{t 0} T\right)
$$

De acuerdo a los resultados del Test de Hausman ${ }^{6}$, la ecuación (2) se estimó mediante un modelo de efectos fijos ${ }^{7}$ y para cada grupo de IMF. También se calculó un Índice de Lerner promedio para cada grupo ${ }^{8}$.

\subsection{ESTIMACIÓN DEL EFECTO DE LA COMPETENCIA SOBRE LA CALIDAD DE CARTERA}

La especificación econométrica para evaluar el efecto de la competencia en la calidad de cartera es la siguiente:

$$
\begin{aligned}
& \text { Morosidad }_{i t}=\beta_{1}+\beta_{2} \text { ciclo }_{t}+\beta_{3} \text { crecimiento_créd }_{i t}+\beta_{4} t \_ \text {interes }_{i t}+ \\
& \beta_{5} \text { rentabilidad }_{i t}+\beta_{6} \text { eficiencia }_{i t}+\beta_{7} \text { competencia }_{i t} \boxplus \beta_{8} \text { crisis }_{t}+\mu_{i t}
\end{aligned}
$$

La variable endógena es la calidad de cartera que se aproxima mediante la tasa de morosidad, mientras que se han considerado además de la competencia como variables explicativas, el ciclo económico, el crecimiento del crédito, la tasa de interés pasiva, la rentabilidad, la eficiencia y una variable dummy para incorporar el efecto de la crisis financiera.

\footnotetext{
6 Ver resultados en el anexo 1.

7 Ver resultados en el anexo 1.

8 El Índice de Lerner promedio se calculó obteniendo los promedios simples por cada año para cada grupo de análisis.
} 
No fue posible incluir la variable nivel de endeudamiento de los agentes por no disponer de un indicador apropiado de la misma. Asimismo, se consideró no incluir el tipo de cambio como variable explicativa de la morosidad, ya que la proporción de créditos denominados en moneda extranjera es reducida en el sistema microfinanciero peruano.

La ecuación (4) se estimó para cada grupo de IMF a través de un modelo de efectos fijos ${ }^{9}$ mediante el Método Generalizado de Momentos $(\mathrm{GMM})^{10}$.

La Tabla 1 presenta las variables y sus indicadores empleados en el análisis cuantitativo.

Tabla 1. Variables e indicadores

\begin{tabular}{|c|c|c|}
\hline \multicolumn{3}{|r|}{ Función de costos } \\
\hline \multicolumn{3}{|l|}{ Variable dependiente: } \\
\hline Costo total & costotal & Costos operativos más costos financieros \\
\hline \multicolumn{3}{|l|}{ Variables explicativas: } \\
\hline Output o producto & output & Créditos totales \\
\hline Precio de la mano de obra & P_trabajo & Gastos administrativos en personal entre el número de empleados \\
\hline Precio del capital & P_capitfis & $\begin{array}{l}\text { Gastos de capital (amortización más depreciación menos } \\
\text { impuestos) entre el valor de los activos fijos netos }\end{array}$ \\
\hline Precio del capital financiero & p_capfin & Gastos financieros entre pasivos totales \\
\hline \multicolumn{3}{|r|}{ Ecuación de morosidad } \\
\hline \multicolumn{3}{|l|}{ Variable dependiente: } \\
\hline Tasa de morosidad & calidcartera & $\begin{array}{l}\text { Créditos atrasados y en cobranza judicial como porcentaje de los } \\
\text { créditos totales }\end{array}$ \\
\hline \multicolumn{3}{|l|}{ Variables explicativas: } \\
\hline Ciclo económico & pbi & Tasa de crecimiento del PBI \\
\hline Crisis financiera $^{11}$ & crisis & $\begin{array}{l}\text { Variable dicotómica que asume el valor de } 1 \text { en el periodo } \\
\text { octubre 2008- diciembre } 2010\end{array}$ \\
\hline Tasa de interés activa & tasaactiva & Ingresos financieros sobre créditos totales \\
\hline Crecimiento del crédito & crec_mercado & Tasa de crecimiento de créditos totales \\
\hline Rentabilidad & roe & ROE \\
\hline Eficiencia & eficiencia & Créditos directos/Número de oficinas (Miles S/.) \\
\hline Competencia & IL & Índice de Lerner \\
\hline
\end{tabular}

\subsection{INFORMACIÓN ESTADÍSTICA}

La información estadística es de naturaleza panel y cubre el periodo enero 2003 a diciembre 2015. La información financiera proviene de las estadísticas publicadas por la SBS mientras que la información macroeconómica de las estadísticas publicadas

9 En el anexo 2 se encuentran los resultados del Test de Hausman para cada grupo de IMF.

${ }^{10}$ En el análisis econométrico se hicieron las pruebas de Multicolinealidad, Heterocedasticidad y Autocorrelación. Los resultados mostraron problemas de heterocedasticidad por lo que se consideró pertinente realizar la regresión con el GMM que brinda resultados más robustos considerando dicho problema.

11 Es necesario aclarar que dado el cambio en el contexto internacional por la crisis financiera de 2008, se consideró adecuado incluir dicha variable para poder capturar su efecto en este análisis. 
por el Banco Central de Reserva del Perú. Se consideró a todas aquellas IMF que tuvieron por lo menos tres años de actividad durante el periodo de análisis. En los casos de fusiones, cada empresa fusionada se ha considerado independiente en el periodo previo a la fusión. El total de entidades microfinancieras incluidas en la muestra es de 42. El panel es desbalanceado porque algunas entidades aparecieron o desaparecieron durante el periodo de estudio. Todas las variables que mostraron un componente estacional fueron desestacionalizadas mediante el método ARIMA X12.

A continuación, se presentan las estadísticas descriptivas de las variables que se utilizan en el análisis.

Tabla 2. Estadísticas descriptivas

\begin{tabular}{|c|c|c|c|c|c|c|c|c|}
\hline Variable & Mean & Std. Dev & Variable & Mean & Std. Dev & Variable & Mean & Std. Dev \\
\hline \multicolumn{3}{|c|}{ GRUPO I } & \multicolumn{3}{|c|}{ GRUPO II } & \multicolumn{3}{|c|}{ GRUPO III } \\
\hline \multicolumn{9}{|c|}{ Análisis de competencia } \\
\hline $\begin{array}{c}\text { p_trabajo } \\
\text { (miles de S/ } \\
\text { al 2009) }\end{array}$ & 4,57 & 1,28 & $\begin{array}{l}\text { p_trabajo } \\
\text { (miles de S/ } \\
\text { al 2009) }\end{array}$ & 3,88 & 1,13 & $\begin{array}{c}\text { p_trabajo } \\
\text { (Miles de S/ } \\
\text { al 2009) }\end{array}$ & 3,69 & 1,42 \\
\hline $\begin{array}{c}\text { P_capitfis } \\
(\%)\end{array}$ & 1,39 & 2,75 & $\begin{array}{c}\text { P_capitfis } \\
(\%)\end{array}$ & 0,32 & 0,14 & $\begin{array}{c}\text { P_capitfis } \\
(\%)\end{array}$ & 0,23 & 0,35 \\
\hline $\begin{array}{c}\text { P_capfinan } \\
(\%)\end{array}$ & 0,085 & 0,03 & $\begin{array}{c}\text { P_capfinan } \\
(\%)\end{array}$ & 0,09 & 0,041 & $\begin{array}{c}\text { P_capfinan } \\
(\%)\end{array}$ & 0,14 & 0,1 \\
\hline $\begin{array}{c}\text { precio } \\
\text { (miles de S/.) }\end{array}$ & 0,56 & 0,24 & $\begin{array}{c}\text { precio } \\
\text { (miles de S/.) }\end{array}$ & 0,51 & 0,2 & $\begin{array}{c}\text { precio } \\
\text { (miles de S/.) }\end{array}$ & 0,31 & 0,21 \\
\hline $\begin{array}{c}\text { costotal } \\
\text { (miles de S/.) }\end{array}$ & 129382,96 & 120631,53 & $\begin{array}{c}\text { costotal } \\
\text { (miles de S/.) }\end{array}$ & 35824,36 & 29784,69 & $\begin{array}{c}\text { costotal } \\
\text { (miles de S/.) }\end{array}$ & 8964,98 & 9137,28 \\
\hline $\begin{array}{c}\text { Output } \\
\text { (miles de S/.) }\end{array}$ & 1415269,00 & 1390634,00 & $\begin{array}{c}\text { Output } \\
\text { (miles de S/.) }\end{array}$ & 248632,29 & 231762,05 & $\begin{array}{c}\text { Output } \\
\text { (miles de S/.) }\end{array}$ & 40561,05 & 41988,98 \\
\hline Cmarg & 0,23 & 0,096 & Cmarg & 0,16 & 0,13 & Cmarg & 0,23 & 0,16 \\
\hline IL & 0,51 & 0,28 & IL & 0,64 & 0,3 & IL & 0,41 & 0,23 \\
\hline \multicolumn{9}{|c|}{ Análisis de calidad de cartera } \\
\hline $\begin{array}{c}\text { calidcartera } \\
(\%)\end{array}$ & 4,89 & 1,58 & $\begin{array}{c}\text { calidcartera } \\
(\%)\end{array}$ & 5,79 & 2,21 & $\begin{array}{c}\text { calidcartera } \\
(\%)\end{array}$ & 8,06 & 6,93 \\
\hline $\begin{array}{l}\mathrm{pbi}_{-} \\
(\%)\end{array}$ & 6,41 & 3,03 & $\begin{array}{l}\mathrm{pbi}_{-} \\
(\%)\end{array}$ & 6,41 & 3,03 & $\begin{array}{l}\mathrm{pbi}_{-} \\
(\%)\end{array}$ & 6,41 & 3,03 \\
\hline $\begin{array}{c}\text { crec_merc } \\
(\%)\end{array}$ & 0,32 & 0,22 & $\begin{array}{c}\text { crec_merc } \\
(\%)\end{array}$ & 0,42 & 0,4 & $\begin{array}{c}\text { crec_merc } \\
(\%)\end{array}$ & 0,34 & 0,31 \\
\hline $\begin{array}{l}\text { tasaactiva } \\
(\%)\end{array}$ & 39,43 & 20,91 & $\begin{array}{l}\text { tasaactiva } \\
(\%)\end{array}$ & 48,31 & 23,69 & $\begin{array}{l}\text { tasaactiva } \\
(\%)\end{array}$ & 51,38 & 34,28 \\
\hline $\begin{array}{l}\text { roe } \\
(\%)\end{array}$ & 21,69 & 18,63 & $\begin{array}{l}\text { roe } \\
(\%)\end{array}$ & 19,43 & 16,87 & $\begin{array}{l}\text { roe } \\
(\%)\end{array}$ & 10,82 & 9,87 \\
\hline eficiencia & 149,63 & 85,21 & eficiencia & 131,87 & 74,38 & eficiencia & 117,39 & 79,68 \\
\hline
\end{tabular}




\section{RESULTADOS}

Los resultados obtenidos en las estimaciones se presentan a continuación considerando los tres grupos de $\mathrm{IMF}^{12}$.

\subsection{Primer grupo: IMF grandes}

Con relación al Índice de Lerner promedio, en el Gráfico 5 se observa que si bien al inicio del periodo de análisis el indicador muestra una tendencia creciente, a partir de mediados del 2004, este sigue un comportamiento decreciente hasta el final del periodo analizado. La trayectoria seguida por el Índice de Lerner revela una reducción del poder de mercado de este primer grupo de operadores microfinancieros consistente, con un aumento de la competencia entre las entidades que conforman este grupo.

\section{Gráfico 5. Índice de Lerner Promedio - Primer grupo}

0.90

0.80

0.70

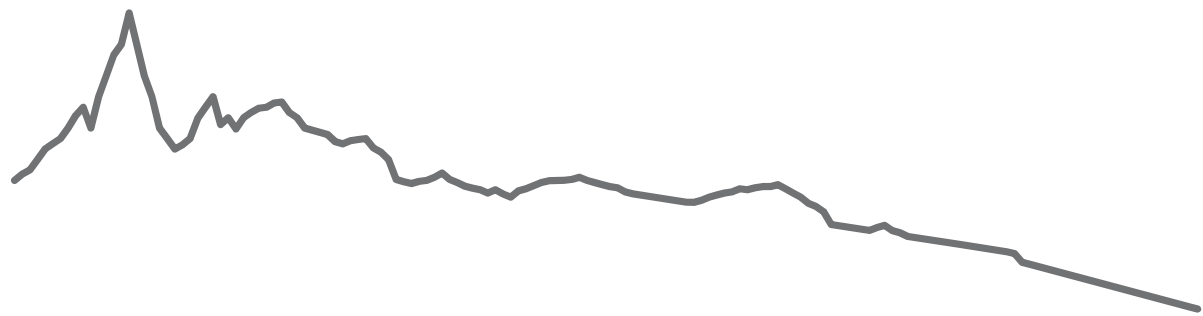

0.50

0.40

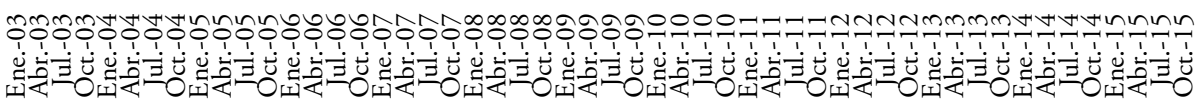

Fuente: SBS. Elaboración: propia.

Este grupo está conformado por instituciones con una gran participación en el mercado microcrediticio. La expansión de las actividades microcrediticias durante la primera década del milenio, que llevó al crecimiento significativo de las entidades que conforman este grupo, así como a su actuación en todo el territorio nacional habrían sido factores que han condicionado una significativa intensificación de la competencia entre estas instituciones.

\footnotetext{
12 Para verificar los resultados encontrados, se considera adecuado realizar este mismo análisis empírico pero dividido por tipo de institución. Dichos resultados se presentarán en el anexo 3 en donde se encuentra la misma relación entre la competencia y la calidad de cartera de los créditos otorgados.
} 
Sobre la relación entre la competencia y la calidad de cartera, los resultados de las estimaciones para este primer grupo de IMF se presenta en la Tabla 3.

Tabla 3. Resultados para el primer grupo

\begin{tabular}{r|c|c}
\hline Variable & Con_crisis & Sin_crisis \\
\hline pbi_ & $\left(-0,81^{* *}\right)$ & $\left(-0,78^{* *}\right)$ \\
crec_cred & $\left(0,12^{*}\right)$ & 0,11 \\
tasaactiva & $\left(0,132^{* *}\right)$ & $\left(0,09^{* *}\right)$ \\
roe & $\left(-0,09^{* *}\right)$ & $\left(-0,07^{* *}\right)$ \\
eficiencia & $\left(-1,14^{* *}\right)$ & $\left(-1,08^{* *}\right)$ \\
IL & $\left(-1,69^{* * *}\right)$ & $\left(-1,73^{* *}\right)$ \\
crisis & $\left(0,88^{* *}\right)$ & \\
_cons & $7,63^{* *}$ & $759^{* * *}$ \\
\hline R2 & 0,8 & 0,72 \\
\hline
\end{tabular}

legend: ${ }^{*} \mathrm{p}<, 01 ; \mathrm{p}^{* *}<, 05 ; \mathrm{p}^{* * *}<, 001$

Se realizaron dos estimaciones, en la primera se consideró el efecto de la crisis financiera y en la segunda se omitió esta variable a fin de verificar la importancia de incorporar el efecto de la crisis financiera en el análisis por medio del cambio en la significancia de los coeficientes estimados. La incorporación del efecto de la crisis financiera en la estimación proporciona un mejor ajuste y coeficientes estimados con significancia estadística y con los signos esperados. El Índice de Lerner presenta un coeficiente estimado con signo negativo, lo que muestra que la disminución del poder de mercado genera un aumento en la tasa de morosidad. Este resultado muestra evidencia de una relación negativa entre la calidad de cartera de las IMF y la competencia en este grupo.

Con respecto a las otras variables incluidas para explicar el comportamiento de la morosidad de los créditos se observa que una mayor tasa de crecimiento del PBI reduce la morosidad crediticia, confirmándose la importancia que tiene el ciclo económico en la explicación del comportamiento de la calidad de la cartera. Una expansión económica reduce la morosidad y una contracción la aumenta. Por otra parte, una mayor tasa de interés incrementa la tasa de morosidad así como una expansión de la propia cartera de créditos. Lo primero podría indicar que un mayor costo del crédito puede estar asociado con el desarrollo de un mayor riesgo moral que conlleva a un deterioro de la cartera crediticia mientras que lo segundo, con un relajamiento en los estándares de riesgo que genera una mayor tasa de morosidad. Mayor rentabilidad y mayor eficiencia contribuyen a reducir la morosidad de las carteras de créditos de estas IMF. Finalmente, la crisis ha contribuido a aumentar la morosidad de los créditos tal como lo muestra el coeficiente estimado con signo positivo y de significancia estadística. 
Los resultados obtenidos sin considerar la variable crisis, son similares en cuanto a los signos de los coeficientes estimados. No obstante, algunos de los coeficientes dejan de ser significativos como el de la tasa de crecimiento de los créditos.

Se concluye, por tanto que en este grupo de IMF, que agrupa a las entidades más grandes del sistema, un aumento de la competencia va de la mano con un aumento en la tasa de morosidad y este resultado se obtiene luego de controlar el efecto de otras variables que también afectan la tasa de morosidad.

\subsection{SEgUNDO GRUPO: IMF MEDIANAS}

En este segundo grupo se encuentran once entidades con volúmenes medianos de cartera de créditos. La evolución del Índice de Lerner promedio para este grupo muestra una tendencia decreciente a lo largo de todo el periodo analizado. Sin embargo, en los últimos meses del estudio, se puede apreciar un leve crecimiento del índice promedio de Lerner (ver Gráfico 6), lo que reflejaría una disminución de la competencia en este grupo en el último ańo del periodo estudiado.

Gráfico 6. Índice de Lerner Promedio - Segundo grupo

0.8

0.7

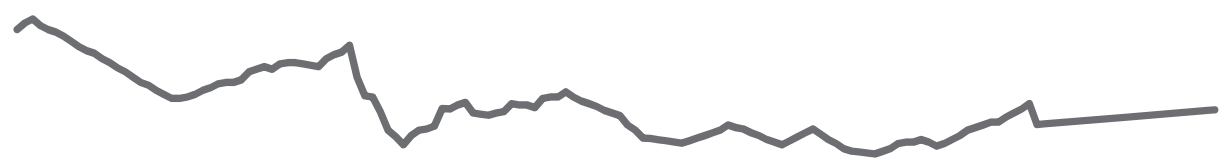

0.6

0.5

0.4

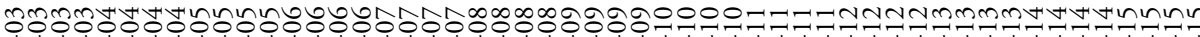

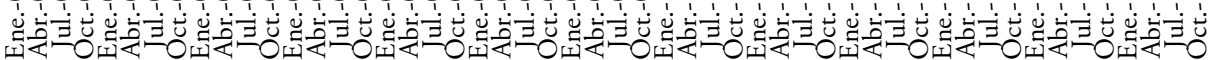

Fuente: SBS. Elaboración propia. 
Los resultados de la estimación de la ecuación (4) se muestran en la Tabla 4:

Tabla 4. Resultados para el segundo grupo

\begin{tabular}{r|c|c}
\hline Variable & Con_crisis & Sin_crisis \\
\hline pbi_ & $\left(-0,76^{* *}\right)$ & $\left(-0,67^{* *}\right)$ \\
crec_cred & $0,09^{*}$ & 0,08 \\
tasaactiva & $0,09^{* *}$ & $0,12^{* *}$ \\
roe & $\left(-0,08^{*}\right)$ & $\left(-0,06^{*}\right)$ \\
eficiencia & $\left(-0,91^{* *}\right)$ & $\left(-0,93^{*}\right)$ \\
IL & $\left(-1,38^{* *}\right)$ & $\left(-1,30^{*}\right)$ \\
crisis & $\left(0,84^{* *}\right)$ & \\
_cons & $3,64^{* *}$ & $3,16^{*}$ \\
\hline R2 & 0,71 & 0,6 \\
\hline
\end{tabular}

legend: ${ }^{*} \mathrm{p}<, 01 ; \mathrm{p}^{* *}<, 05 ; \mathrm{p}^{* * *}<, 001$

En la primera estimación, la que incorpora el efecto de la crisis financiera, todos los coeficientes estimados son significativos y presentan los signos esperados y cuenta con un mejor ajuste con relación a la segunda estimación que no incluye la variable crisis.

El coeficiente estimado, asociado al Índice de Lerner, es negativo y estadísticamente significativo, evidenciando que un mayor poder de mercado genera una menor tasa de morosidad (y viceversa) en este segundo grupo de IMF. Es decir, que la mayor competencia en el mercado deteriora la calidad de cartera de créditos en este grupo de IMF.

Respecto a los otros determinantes de la calidad de cartera, se encuentra que el ciclo económico es un factor importante para explicar la morosidad en este grupo. Este resultado se encuentra, tanto cuando se evalúa el efecto de la crisis como cuando no se considera este efecto, y ambos casos, un ciclo expansivo reduce la morosidad y un ciclo recesivo la aumenta. La expansión de los créditos solo juega un rol importante para explicar la tasa de morosidad en este grupo, cuando se considera el efecto de la crisis. Como era de esperarse, una tasa activa elevada incrementa la morosidad en ambas ecuaciones, mientras que cuanto más eficientes son las IMF poseen menores tasas de morosidad. Igualmente, cuanto más rentables, sus niveles de morosidad son menores.

Por tanto, los resultados muestran evidencia que en este conjunto de IMF de tamaño mediano la competencia genera un deterioro en la calidad de cartera crediticia.

\subsection{TERCER GRUPO: IMF PEQUEÑAS}

En este grupo, el Índice de Lerner promedio sigue una tendencia creciente hasta mediados del 2010 aproximadamente, y luego empieza a decrecer hasta el final del periodo de análisis (ver Gráfico 7). La reducción del poder de mercado exhibida en este grupo de IMF es consistente con un aumento de la competencia entre las entidades de este grupo. 


\section{Gráfico 7. Índice de Lerner Promedio - Tercer grupo}

0.5

0.4

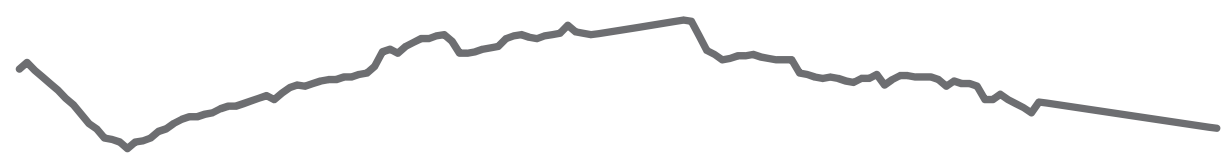

0.3

0.1

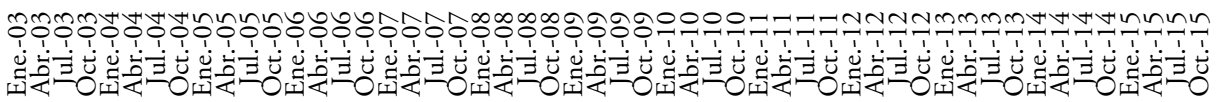

Fuente: SBS. Elaboración propia.

La Tabla 5, presenta los resultados de la estimación de los efectos de la competencia sobre la calidad de cartera.

Tabla 5. Resultados para el tercer grupo

\begin{tabular}{r|c|c}
\hline Variable & Con_crisis & Sin_crisis \\
\hline pbi_ & $\left(-0,70^{* *}\right)$ & $0,61^{*}$ \\
crec_cred & $0,08^{*}$ & $0,03^{*}$ \\
tasaactiva & $0,09^{* * *}$ & $0,07^{* *}$ \\
roe & $\left(-0,07^{*}\right)$ & $\left(-0,04^{*}\right)$ \\
eficiencia & $\left(-0,81^{*}\right)$ & $\left(-0,77^{*}\right)$ \\
IL & $\left(-1,26^{* * *}\right)$ & $\left(-1,19^{* *}\right)$ \\
crisis & $\left(-0,89^{* *}\right)$ & \\
_cons & $2,69^{* *}$ & $2,57^{* *}$ \\
\hline R2 & 0,69 & 0,71 \\
\hline
\end{tabular}

legend: ${ }^{*} \mathrm{p}, .01 ; \mathrm{p}^{* *}<, 05 ; \mathrm{p}^{* * *}<, 001$

En ambas ecuaciones (con y sin la variable crisis), la mayoría de los coeficientes estimados son significativos y tienen los signos esperados. Las estimaciones muestran que el efecto de la competencia sobre la morosidad es negativo, es decir, que cuando el incremento de la competencia deteriora la calidad de cartera de las IMF de menor tamaño en el mercado microfinanciero peruano. 
Nuevamente se encuentra que en ciclos expansivos de la actividad económica, la morosidad crediticia disminuye y en ciclos depresivos, aumenta. Una mayor tasa de interés activa eleva la morosidad de los créditos, de la misma manera que una expansión de las colocaciones. Asimismo, cuanto más rentable y eficiente la institución, menor es la morosidad de su cartera crediticia. Para este grupo de IMF, la crisis ha generado un efecto negativo en la morosidad, resultado que no es consistente con lo esperado, dado que la crisis como shock negativo debería generar mayores niveles de incumplimiento por parte de los agentes, reflejándose en mayores niveles de morosidad.

En resumen, los resultados hallados evidencian que tanto para el grupo de IMF con grandes volúmenes promedio de colocaciones, como para las que tienen un menor promedio de créditos, el poder de mercado se ha reducido en el periodo analizado, lo que revelaría que en los mercados donde operan estas entidades la competencia se ha intensificado. En el grupo de entidades de tamaño mediano, a pesar de que el Índice de Lerner inicialmente reflejan un comportamiento decreciente, al final del periodo analizado presenta una ligera tendencia creciente, lo que indica un aumento del poder de mercado en este grupo de IMF y, por ende, una reducción de la competencia entre este grupo de operadores microfinancieros. También se ha encontrado evidencia de que la competencia tiene un efecto negativo sobre la calidad de cartera crediticia, reflejado en el crecimiento de la tasa de morosidad. Este resultado es generalizable a las IMF de todo tamańo, es decir, es un resultado que se aplica a todo el mercado microcrediticio peruano. Y además, este resultado se obtiene controlando el comportamiento de otras variables importantes para explicar el desarrollo de la morosidad microcrediticia como son el ciclo económico, la expansión de los créditos, la eficiencia y la rentabilidad de las instituciones, además del efecto de la crisis financiera internacional de 2008.

\section{CONCLUSIONES}

De la literatura económica se desprende que el sector financiero se caracteriza por alejarse de la competencia perfecta, debido a las fallas que presenta este sector. No obstante, las características actuales de este mercado en el Perú muestran un mayor dinamismo competitivo.

Por lo tanto, a pesar de que una mayor competencia suele significar mayores beneficios para los agentes que participan en el mercado, no existe consenso respecto a los efectos de la competencia en la calidad de cartera de créditos otorgados. Un primer enfoque argumenta que una mayor competencia deteriora la calidad de cartera, mientras que el segundo enfoque considera lo contrario; es decir que a mayor competencia mejorará la calidad de la cartera crediticia.

Ante esta discrepancia teórica, el objetivo de la presente investigación fue analizar el vínculo existente entre la competencia y la calidad de cartera de colocaciones de las entidades microfinancieras peruanas en el periodo 2003-2015. Como indicador de 
competencia se empleó el Índice de Lerner y como indicador de calidad de cartera, la tasa de morosidad. El análisis se realizó segmentando el sector microfinanciero en tres grupos de IMF (grandes, medianas y pequeñas) de acuerdo al volumen promedio de colocaciones. Esto con el fin de tomar en cuenta la heterogeneidad que existe entre las entidades microfinancieras peruanas.

Los resultados encontrados permiten inferir que la competencia ha aumentado tanto para el grupo de IMF de mayor tamaño como para las IMF más pequeñas ya que el Índice de Lerner tiene una tendencia decreciente durante el periodo de análisis. La situación es distinta respecto al segundo grupo, debido a que si bien el comportamiento del Índice de Lerner en casi todo el periodo de análisis presenta una tendencia decreciente, se revierte en los últimos ańos para mostrar una ligera tendencia creciente.

Con relación al efecto de la competencia sobre la calidad de cartera microcrediticia, los resultados encontrados permiten concluir que una mayor competencia está relacionada negativamente con la calidad de cartera. Es decir, se ha observado que existe una relación inversa entre el Índice de Lerner y la tasa de morosidad en el mercado microfinanciero peruano, durante el periodo estudiado. Este resultado, se encuentra en los tres grupos de IMF estudiados y controlando el comportamiento de otras variables de carácter micro y macroeconómico que afectan la calidad de cartera como por ejemplo, el ciclo económico, la expansión del crédito, la tasa de interés activa, la rentabilidad, la eficiencia y la crisis financiera internacional. La relación negativa de la competencia sobre la calidad de cartera en el mercado microfinanciero peruano en el periodo 20032015 es consistente con el enfoque teórico según el cual, la competencia deteriora la estabilidad financiera de las entidades a través de un incremento en el riesgo crediticio de sus colocaciones.

Por otro lado, se espera que en futuras investigaciones se pueda incorporar a la banca tradicional en el análisis de la competencia en el mercado microfinanciero dada su participación (aunque decreciente) en este sector.

Finalmente, ante los resultados obtenidos en esta investigación y los cambios que vienen ocurriendo en el sector microfinanciero peruano, es fundamental llamar la atención de las instituciones comprometidas con la marcha del sector en términos de supervisión y regulación, para que a través del rol garanticen que la competencia siga beneficiando a los consumidores de este mercado y a su vez, se desarrolle de manera saludable para la estabilidad financiera de los intermediarios microfinancieros. 


\section{ANEXOS}

Anexo 1. Resultados de la función de costos translogarítmica

\begin{tabular}{|c|c|c|c|c|c|}
\hline \multicolumn{2}{|c|}{ PRIMER GRUPO } & \multicolumn{2}{|c|}{ SEGUNDO GRUPO } & \multicolumn{2}{|c|}{ TERCER GRUPO } \\
\hline Variable & FunCT & Variable & FunCT & Variable & FunCT \\
\hline lnp_capfin & $3,83^{* * *}$ & lnp_capfin & $4,0^{* *}$ & lnp_capfin & $2,98^{* * *}$ \\
\hline lnp_trabajo & $210^{* * *}$ & lnp_trabajo & $4,05^{* * *}$ & lnp_trabajo & $3,87^{* *}$ \\
\hline lnp_capifis & $2,09^{* *}$ & lnp_capifis & $0,55^{* *}$ & lnp_capifis & $3,09^{* * *}$ \\
\hline mitadlnp_capfin & $0,45^{* *}$ & mitadlnp_capfin & $0,19^{*}$ & mitadlnp_capfin & $0,32^{* *}$ \\
\hline mitadlnp_trabajo & $0,30^{* *}$ & mitadlnp_trabajo & $104^{* * *}$ & mitadlnp_trabajo & $0,27^{* *}$ \\
\hline mitadlnp_capifis & $0,05^{*}$ & mitadlnp_capifis & $0,08^{* *}$ & mitadlnp_capifis & $011^{* * *}$ \\
\hline mitadlnp_capfin 2 & $\left(-0,90^{*}\right)$ & mitadlnp_capfin2 & $\left(-1,03^{* *}\right)$ & mitadlnp_capfin2 & $\left(-0,20^{* *}\right)$ \\
\hline mitadlnp_trabajo2 & $0,14^{*}$ & mitadlnp_trabajo2 & $\left(-0,31^{*}\right)$ & mitadlnp_trabajo2 & $0,37^{* *}$ \\
\hline mitadlnp_capifis2 & $\left(-0,88^{*}\right)$ & mitadlnp_capifis2 & $\left(-0,02^{* *}\right)$ & mitadlnp_capifis2 & $\left(-0,64^{* * *}\right)$ \\
\hline Inoutput & $0,49^{* * *}$ & lnoutput & 1,6 & lnoutput & $1,36^{* *}$ \\
\hline mitadlnoutput2 & $0,087^{* *}$ & mitadlnoutput2 & $0,07^{* *}$ & mitadlnoutput2 & $014^{* * *}$ \\
\hline Inoutptra & $\left(-0,21^{* *}\right)$ & lnoutptra & $\left(-1,0^{* *}\right)$ & lninptra & $\left(-1,66^{* * *}\right)$ \\
\hline Inoutpcapfin & $011^{* * *}$ & lnoutpcapfin & $0,12^{*}$ & lninpcapfin & $\left(-0,15^{* *}\right)$ \\
\hline Inoutpcapfis & $0,09^{* * *}$ & lnoutpcapfis & $0,04^{*}$ & lninpcapfis & $\left(-0,14^{* * *}\right)$ \\
\hline tenden & $\left(-0,15^{* * *}\right)$ & tenden & $\left(-0,10^{* *}\right)$ & tenden & $\left(-0,05^{* *}\right)$ \\
\hline mitadtende2 & 0,01 & mitadtende2 & $0,01^{*}$ & mitadtende2 & $0,00^{*}$ \\
\hline outputend & $\left(-0,02^{* *}\right)$ & outputend & $\left(-0,03^{*}\right)$ & outputend & $\left(-0,08^{* *}\right)$ \\
\hline tendenlnp_capfin & $\left(-0,01^{*}\right)$ & tendenlnp_capfin & $\left(-0,02^{* *}\right)$ & tendenlnp_capfin & $\left(-0,04^{* *}\right)$ \\
\hline tendenlnp_trabajo & $\left(-0,07^{* *}\right)$ & tendenlnp_trabajo & $\left(-0,06^{* *}\right)$ & tendenlnp_trabajo & $\left(-0,03^{* *}\right)$ \\
\hline tendenlnp_capifis & $\left(-0,01^{* *}\right)$ & tendenlnp_capifis & $\left(-0,10^{* *}\right)$ & tendenlnp_capifis & $\left(-0,05^{* * *}\right)$ \\
\hline const & $\left(-1,65^{*}\right)$ & const & $\left(-2,66^{* *}\right)$ & const & 7,02 \\
\hline $\mathrm{R} 2$ & 0,76 & R2 & 0,81 & $\mathrm{R} 2$ & 0,69 \\
\hline Test Hausman & 408,00 & Test Hausman & 260,05 & Test Hausman & 328,01 \\
\hline Prob $>$ Chi2 & 0.00 & Prob $>$ Chi 2 & 0.00 & Prob $>$ Chi2 & 0.00 \\
\hline \multicolumn{6}{|c|}{ legend: ${ }^{*} \mathrm{p}<0,1 ;{ }^{* *} \mathrm{p}<0,05 ;{ }^{* *} \mathrm{p}<0,001$} \\
\hline
\end{tabular}


Anexo 2. Test de Hausman regresión Calidad de cartera

PRIMER GRUPO

\begin{tabular}{r|c|c}
\hline \multicolumn{1}{c|}{ Variable } & Con_crisis & Si n_cri s s \\
\hline pbi_- & $\left(-0,84923^{* *}\right)$ & $\left(-0,79663^{* * *}\right)$ \\
crec_cred & $\left(0,09245^{*}\right)$ & 0,09022 \\
tasaactiva & $\left(0,124646^{* *}\right)$ & $\left(0,09789^{* *}\right)$ \\
roe & $\left(-0,076514^{* *}\right)$ & $\left(-0,064791^{* *}\right)$ \\
eficiencia & $\left(-1,082719^{* * *}\right)$ & $\left(-1,00748^{* *}\right)$ \\
IL & $\left(-1,64716^{* * *}\right)$ & $\left(-1,60757^{* *}\right)$ \\
crisis & $\left(0,86126^{* *}\right)$ & \\
_cons & $7,58432^{* *}$ & $7,8501^{* * *}$ \\
\hline R2 & 0,854 & 0,793 \\
Test Hausman & 473 & 551 \\
Prob>Chi2 & 0,00 & 0,00 \\
\hline
\end{tabular}

SEGUNDO GRUPO

\begin{tabular}{r|c|c}
\hline \multicolumn{1}{c|}{ Variable } & Con_crisis & Si n_c risis \\
\hline pbi_ & $\left(-0,69738^{* *}\right)$ & $\left(-0,58163^{* * *}\right)$ \\
crec_cred & $\left(0,068342^{*}\right)$ & 0,061436 \\
tasaactiva & $\left(0,09869^{* *}\right)$ & $\left(0,087537^{* *}\right)$ \\
roe & $\left(-0,05879^{*}\right)$ & $\left(-0,04682^{*}\right)$ \\
eficiencia & $\left(-0,9319^{* *}\right)$ & $\left(-0,8863^{*}\right)$ \\
IL & $\left(-1,369402^{* *}\right)$ & $\left(-1,330485^{*}\right)$ \\
crisis & $\left(0,79542^{* *}\right)$ & \\
cons & $3,87035^{* *}$ & $3,012763^{*}$ \\
\hline R2 & 0,786 & 0,635 \\
Test Hausman & 251 & 382 \\
Prob>Chi2 & 0,00 & 0,00 \\
\hline
\end{tabular}

TERCER GRUPO

\begin{tabular}{r|c|c}
\hline \multicolumn{1}{c|}{ Variable } & Con_crisis & Si $\mathbf{n} \_$cri s s \\
\hline pbi_ & $\left(-0,749642^{* *}\right)$ & $\left(0,65867^{*}\right)$ \\
crec_cred & $\left(0,058487^{*}\right)$ & $\left(0,049779^{*}\right)$ \\
tasaactiva & $\left(0,084688^{* * *}\right)$ & $\left(0,0803207^{* *}\right)$ \\
roe & $\left(-0,066905^{*}\right)$ & $\left(-0,05887^{*}\right)$ \\
eficiencia & $\left(-0,88174^{*}\right)$ & $\left(-0,79748^{*}\right)$ \\
IL & $\left(-1,29858^{* * *}\right)$ & $\left(-1,16597^{* *}\right)$ \\
crisis & $\left(-0,86531^{* * *}\right)$ & \\
_cons & $2,730284^{* *}$ & $2,50752^{* *}$ \\
\hline R2 & 0,827 & 0,735 \\
Test Hausman & 357 & 302 \\
Prob>Chi2 & 0,00 & 0,00 \\
\hline
\end{tabular}




\section{Anexo 3}

Resultados para las CMAC

\begin{tabular}{r|c|c}
\hline Variable & Con_crisis & Sin_crisis \\
\hline pbi_ & $\left(-0,687^{*}\right)$ & $\left(-0,718^{* *}\right)$ \\
crec_cred & $0,17^{* *}$ & 0,23 \\
tasaactiva & 0,06 & $0,12^{* *}$ \\
roe & $\left(-0,04^{*}\right)$ & $\left(-0,051^{*}\right)$ \\
eficiencia & $\left(-0,02^{* * *}\right)$ & $\left(-0,14^{*}\right)$ \\
IL & $\left(-1,87^{* *}\right)$ & $\left(-1,47^{*}\right)$ \\
crisis & $\left(0,81^{* *}\right)$ & \\
ccons & $4,28^{*}$ & $2,47^{*}$ \\
\hline $\mathrm{R} 2$ & 0,62 & 0,75 \\
\hline
\end{tabular}

legend: ${ }^{*} \mathrm{p}<, 01 ; \mathrm{p}^{* *}<, 05 ; \mathrm{p}^{* * *}<, 001$

Resultados para las edpymes

\begin{tabular}{r|c|c}
\hline Variable & Con_crisis & Sin_crisis \\
\hline pbi_ & $\left(-0,424^{*}\right)$ & $\left(-0,537^{* *}\right)$ \\
crec_cred & $0,34^{* *}$ & $0,59^{* *}$ \\
tasaactiva & $0,161^{* * *}$ & $0,348^{*}$ \\
roe & $\left(-0,264^{*}\right)$ & $\left(-0,198^{*}\right)$ \\
eficiencia & $\left(-1,06^{* *}\right)$ & $\left(-0,283^{*}\right)$ \\
IL & $\left(-0,58^{* * *}\right)$ & $\left(-1,82^{*}\right)$ \\
crisis & $\left(0,832^{* * *}\right)$ & \\
_cons & $6,21^{*}$ & $7,83^{*}$ \\
\hline R2 & 0,575 & 0,61 \\
\hline
\end{tabular}

legend: ${ }^{*} \mathrm{p}<, 01 ; \mathrm{p}^{* *}<, 05 ; \mathrm{p}^{* * *}<, 001$
Resultados para las CRAC

\begin{tabular}{r|c|c}
\hline Variable & Con_crisis & Si _cris i s \\
\hline pbi_ & $\left(-0,76^{* *}\right)$ & $\left(-0,88^{*}\right)$ \\
crec_cred & $\left(0,19^{* *}\right)$ & $0,246^{*}$ \\
tasaactiva & $\left(0,214^{*}\right)$ & $\left(0,481^{* *}\right)$ \\
roe & $\left(-0,11^{*}\right)$ & $\left(-0,17^{* * *}\right)$ \\
eficiencia & $\left(-1,28^{* *}\right)$ & $\left(-2,08^{* *}\right)$ \\
IL & $\left(-1,47^{* *}\right)$ & $\left(-1,89^{* *}\right)$ \\
crisis & $\left(0,76^{* *}\right)$ & \\
_cons & $6,34^{* *}$ & $8,49^{* *}$ \\
\hline R2 & 0,71 & 0,69 \\
\hline
\end{tabular}

legend: ${ }^{*} \mathrm{p}<, 01 ; \mathrm{p}^{* *}<, 05 ; \mathrm{p}^{* * *}<, 001$

Resultados para las financieras

\begin{tabular}{r|c|c}
\hline Variable & Con_crisis & Sin_crisis \\
\hline pbi_ & $\left(-0,714^{* * *}\right)$ & $\left(-0,624^{* *}\right)$ \\
crec_cred & $0,47^{* *}$ & $0,45^{*}$ \\
tasaactiva & $0,092^{* *}$ & $0,28^{* *}$ \\
roe & $\left(-0,14^{*}\right)$ & $\left(-0,018^{*}\right)$ \\
eficiencia & $\left(-0,08^{* *}\right)$ & $\left(-0,53^{* *}\right)$ \\
IL & $\left(-1,51^{* * *}\right)$ & $\left(-1,73^{*}\right)$ \\
crisis & $\left(0,94^{* *}\right)$ & \\
_cons & $7,02^{*}$ & $4,07^{*}$ \\
\hline R2 & 0,65 & 0,59 \\
\hline
\end{tabular}

legend: ${ }^{\mathrm{p}}<, 01 ; \mathrm{p}^{* *}<, 05 ; \mathrm{p}^{* * *}<, 001$

Resultados para la banca especializada

\begin{tabular}{r|c|c}
\hline Variable & Con_crisis & Sin_crisis \\
\hline pbi_ & $\left(-0,62^{*}\right)$ & $(-0,7)$ \\
crec_cred & $0,451^{* * *}$ & $0,76^{*}$ \\
tasaactiva & $0,29^{* *}$ & $0,314^{*}$ \\
roe & $(-0,417)$ & $\left(-0,251^{* *}\right)$ \\
eficiencia & $\left(-1,68^{* *}\right)$ & $\left(-0,3^{*}\right)$ \\
IL & $\left(-0,87^{* *}\right)$ & $\left(-1,02^{*}\right)$ \\
crisis & $\left(0,72^{*}\right)$ & \\
_cons & $4,321^{*}$ & $3,94^{*}$ \\
\hline R2 & 0,591 & 0,627 \\
\hline
\end{tabular}

legend: ${ }^{*} \mathrm{p}<, 01 ; \mathrm{p}^{* *}<, 05 ; \mathrm{p}^{* * *}<, 001$ 


\section{REFERENCIAS BIBLIOGRÁFICAS}

Aguilar, Giovanna (2014). «Dos estudios sobre las microfinanzas en el Perú». Tesis de doctorado. Pontificia Universidad Católica del Perú, Escuela de Posgrado.

Aguilar Giovanna y Gonzalo Camargo (2004). Análisis de la morosidad de las instituciones microfinancieras (IMF) en el Perú. Lima: IEP.

Allen, Franklin y Douglas Gale (2004). Competition and Financial Stability. Journal of Money, Credit and Banking, 36(3), 453-480. https://doi.org/10.1353/mcb.2004.0038

Aparicio, Carlos, José Gutiérrez, Miguel Jaramillo y Hesiona Moreno (2013). Indicadores alternativos de riesgo de crédito en el Perú: matrices de transición crediticia condicionadas al ciclo económico. Documentos de Trabajo 01-2013. Lima: Superintendencia de Banca, Seguro y Administradoras Privadas de Fondos de Pensiones.

Assefa, Esubalew, Niels Hermes y Aljar Meesters (2013). Competition and the Performance of Microfinance Institutions. Applied Financial Economics, Taylor \& Francis Journals, 23(9), 767-782. https://doi.org/10.1080/09603107.2012.754541

Bangia, Anil, Francis Diebold y Til Schuermann (2002). Ratings Migration and the Business Cycle, With Applications to Credit Portfolio Stress Testings. Journal of Bankings \& Finance, 26, 445-474. https://doi.org/10.1016/S0378-4266(01)00229-1

Barrón, Pavel (2011). El efecto del tamaño y la concentración del mercado bancario sobre el riesgo sistémico de la banca privada boliviana. Revista de Análisis, 15, 97-143. Bolivia.

Beck, Thorsten (2008). «Bank Competition and Financial Stability: Friends or Foes?» World Bank. Written for the G20 Seminar on Competition in the Financial Sector, February 2008. https://doi.org/10.1596/1813-9450-4656

Beck, T., Demirguc-Kunt, A. y R. Levine, R. (2006) Bank concentration, competition, and crises: first results. Journal of Banking and Finance, 30, 1581-1603.

Bell, Frederick y Neil Murphy (1968). Economies of Scale and Division of Labor in Commercial Banking. Southern Economic Journal, 35(2), 131-139. https://doi.org/10.2307/1056322

Benston, George (1965). Branch Banking and Economies of Scale. Journal of Finance, 20, 312-331. https://doi.org/10.1111/j.1540-6261.1965.tb00212.x

Benston, George, Gerald Hanweck y David Humphrey (1982). Scale Economies in Banking: A Restructuring and Reassessment. Journal of Money, Credit and Banking, 14(4), 435-456. https://doi.org/10.2307/1991654

Berger, A., L. Klapper y R. Turk-Ariss (2009). Bank Competition and Financial Stability. Journal of Financial Services Research, 35(2), 99-118. https://doi.org/10.1007/s10693008-0050-7

Boone, Jan, Jan Van Ours y Henry van der Wiel (2007). How (not) to measure competition. La Haya: CPB Nertherlands Bureau for Economic Policy Analysis.

Boyd, John y Gianni de Nicolo (2005). The Theory of Bank Risk-Taking and Competition Revisited. Journal of Finance, 60(3), 1329-1343. https://doi.org/10.1111/ j.1540-6261.2005.00763.x

Caminal, Ramon y Carmen Matutes (2002). Market Power and Banking Failures. International Journal of Industrial Organization, 20, 1341-1361. https://doi.org/10.1016/S01677187(01)00092-3

Cetorelli, Nicola y Pietro Peretto (2000). Oligopoly Banking and Capital Accumulation. Federal Reserve Bank of Chicago. Working Paper Series WP-0-12. Disponible en http://www. chicagofed.org/digital_assets/publications/working_papers/2000/wp2000_12.pdf 
Conger Lucy, Patricia Inga y Richard Webb (2009). El árbol de la mostaza: historia de las microfinanzas en el Perú. Lima: Instituto del Perú de la Universidad de San Martín de Porres.

Del Pozo, César (2011). «Nivel de competencia en las microfinanzas, evidencia para el Perú». XXVI Encuentro de Economistas del BCRP. Lima: Banco Central de Reserva del Perú.

Díaz, Oscar (2009). Determinantes del ratio de morosidad en el sistema financiero boliviano. La Paz: Banco Central de Bolivia.

Gehrig, Thomas (1998). Screening, Cross-Border Banking and the Allocation of Credit. Research in Economics, 52, 387-407. https://doi.org/10.1006/reec.1998.0175

Hauswald, Robert y Robert Márquez (2003). Information Technology and Financial Services Competition. Review of Financial Studies, 16(3), 921-948. https://doi.org/10.1093/rfs/ hhg017

Hellmann, Thomas, Kevin Murdock y Joseph Stiglitz (2000). Liberalization, Moral Hazard in Banking and Prudential Regulation: Are Capital Requirements Enough? The American Economic Review, 90, 147-165. https://doi.org/10.1257/aer.90.1.147

Jiménez, Gabriel, Jose Lopez y Jesús Saurina (2007). How does competition impact bank risktaking? Federal Reserve Bank of San Francisco. Working Paper Series 2007-23. https:// doi.org/10.2139/ssrn.965462

Jones, Matthew (2005). Estimating Markov Transition Matrices Using Proportions Data: An application to Credit Risk. IMF Working Paper. WP/05/2019. https://doi. org/10.5089/9781451862386.001

Kumar, Kar y Rajuela Bali (2014). Competition, Performance and Portfolio Quality in Microfinance Markets: A Study Using Global Panel Data. Working Paper Series from Uppsala University.

Keeton William y Charles Morris (1987). Why do Banks' Loan Losses Differ? Economic Review: Federal Reserve Bank of Kansas City, mayo, 3-21.

Maudos, Joaquín; Juan Fernández y Francisco Pérez (2002). Market Power in European Banking Sector. Working Papers, serie EC 2002-05. Instituto Valenciano de Investigaciones Económicas.

Maudos, Joaquín y Juan Fernández (2010). Dimensión bancaria, poder de mercado y estabilidad financiera. Perspectivas del Sistema Financiero, 99, 69-81. Valencia.

Murrugarra, Eduardo y Alfredo Ebentreich (1999). Determinantes de morosidad en entidades de microfinanzas: evidencia de las EDPYMEs. Documentos de Trabajo 03-1999. Lima: Superintendencia de Banca, Seguro y Administradoras Privadas de Fondos de Pensiones.

Pérez, Luisa (2009). "Competencia y estabilidad en el sistema bancario: evidencia para la República Dominicana». Tesis de magíster. Pontificia Universidad Católica de Chile.

Petersen, Mitchel y Raughu Rajan (1995). The Effect of Credit Market Competition on Lending Relationship. Quarterly Journal of Economics, 110, 407-443. https://doi. org/10.2307/2118445

Shaeck, Klaus, Martin Cihak y Simon Wolfe (2009). Are Competition Banking Systems More Stable? Journal of Money, Credit and Banking, 41, 711-734. https://doi.org/10.1111/ j.1538-4616.2009.00228.x

Sinkey, Joseph y Mary Greenawalt (1991). Loan-loss Experience and Risk-taking Behavior at Large Commercial Banks. Journal of Financial Services Research, 5, 43-59. https://doi. org/10.1007/BF00127083

Stiglitz, Joseph y Andrew Welss (1981). Credit Rationing in Markets with Imperfect Information. The American Economic Review, 71(3), 393-410. 
Talledo, Jacqueline (2014). La morosidad de la cartera de créditos a la microempresa de las cajas rurales de ahorro y crédito y su relación con la competencia. Documento de Trabajo SBS DT/01/2014. Lima: SBS.

Turk, Rima (2010). On the Implications of Market Power in Banking: Evidence from Developing Countries. Journal of Banking \& Finance, 34, 765-775. https://doi.org/10.1016/j. jbankfin.2009.09.004

Vela, Lindon y Javier Uriol (2012). «Los factores que determinan la calidad de cartera crediticia de las entidades microfinancieras de la Amazonía peruana en el periodo 2008-2011». Documento ganador en el Concurso Nacional de Investigación Económica en el marco del Congreso de Estudiantes de Economía 2012.

Vives, Xavier (2014). La politica de competencia en el sector bancario. México: Centro Regional de Competencia (CRC) para América Latina.

Documento recibido el 20 de enero de 2016 y aprobado el 7 de noviembre de 2016 